\title{
A Polynomial Time Nilpotence Test for Galois Groups and Related Results
}

\author{
V. Arvind \\ Institute of Mathematical Sciences \\ C.I.T Campus,Chennai, India 600113 \\ arvind@imsc.res.in \\ Piyush P Kurur \\ Department of Computer Science and Engineering, \\ Indian Institute of Technology, Kanpur, \\ Kanpur, UP 208016, India \\ ppk@cse.iitk.ac.in *
}

\begin{abstract}
We give a deterministic polynomial-time algorithm to check whether the Galois group $\mathrm{Gal}(f)$ of an input polynomial $f(X) \in \mathbb{Q}[X]$ is nilpotent: the running time is polynomial in size $(f)$. Also, we generalize the LandauMiller solvability test to an algorithm that tests if $\operatorname{Gal}(f)$ is in $\Gamma_{d}$ : this algorithm runs in time polynomial in size $(f)$ and $n^{d}$ and, moreover, if $\operatorname{Gal}(f) \in \Gamma_{d}$ it computes all the prime factors of \#Gal $(f)$.
\end{abstract}

\section{Introduction}

Computing the Galois group of a polynomial is a fundamental problem in algorithmic number theory. Asymptotically, the best known algorithm is due to Landau [3]: on input $f(X)$, it takes time polynomial in size $(f)$ and the order of its Galois group Gal $(f)$. If $f(X)$ has degree $n$ then Gal $(f)$ can have $n$ ! elements. Thus, Landau's algorithm takes time exponential in input size. It is a long standing open problem if there is an asymptotically faster algorithm for computing Gal $(f)$. Lenstra's survey [6] discusses this and related problems.

A different kind of problem is to test for a given $f(x)$ if Gal $(f)$ satisfies a specific property without explicitly computing it. Galois's seminal work showing $f(X)$ is solvable by radicals if and only if $\mathrm{Gal}(f)$ is solvable is a classic example. Landau and Miller [4] gave a remarkable polynomial-time algorithm for testing solvability of the Galois group without computing the Galois group.

* work done when the author was a PhD student at the Institute of Mathematical Sciences, Chennai. 


\subsection{The results of this article}

Our main result is a deterministic polynomial-time algorithm for testing if $\mathrm{Gal}(f)$ is nilpotent. Although nilpotent groups are a proper subclass of solvable groups, the Landau-Miller solvability test does not give a nilpotence test. Basically, the Landau-Miller test is a method of testing that all composition factors of $\operatorname{Gal}(f)$ are abelian, which tests solvability. Nilpotence however is a more "global" property, in the sense that it cannot be inferred by properties of the composition factors alone.

We note here that nilpotence testing of Galois groups has been addressed by other researchers with the goal of developing good practical algorithms. For example in 22 an algorithm for nilpotence testing is given which takes time polynomial in size $(f)$ and \# Gal $(f)$. However, ours is the first algorithm that is provably polynomial time, i.e. runs in time polynomial in size $(f)$, on all inputs.

Next, we show that the Landau-Miller solvability test can be extended to a polynomial-time algorithm for checking, given $f \in \mathbb{Q}[X]$, if Gal $(f)$ is in $\Gamma_{d}$ for constant $d$. A group $G$ is in $\Gamma_{d}$ if there is a composition series $G=G_{0} \triangleright \ldots \triangleright G_{t}=$ $\{1\}$ such that each nonabelian composition factor $G_{i} / G_{i+1}$ is isomorphic to a subgroup of $S_{d}$. The class $\Gamma_{d}$ often arises in permutation group algorithms (see e.g. 7]). Moreover, if $\operatorname{Gal}(f) \in \Gamma_{d}$, the prime factors of $\# \operatorname{Gal}(f)$ can be found in polynomial time.

\subsection{Galois theory overview}

We quickly recall some Galois theory (see, e.g. 5 for details). Let $L$ and $K$ be fields. If $L \supset K$, we say that $L$ is an extension of $K$ and denote it by $L / K$. If $L / K$ then $L$ is a vector space over $K$ and by the degree of $L / K$, denoted by $[L: K]$, we mean its dimension. An extension $L / K$ is finite if its degree $[L: K]$ is finite. If $L / M$ and $M / K$ are finite extensions then $[L: K]=[L: M] \cdot[M: K]$. The polynomial ring $K[X]$ is a unique factorisation domain: every polynomial can be uniquely (up to scalars) written as a product of irreducible polynomials.

Let $L / K$ be an extension. An $\alpha \in L$ is algebraic over $K$ if $f(\alpha)=0$ for some $f(X) \in K[X]$. For $\alpha$ algebraic over $K$, the minimal polynomial of $\alpha$ over $K$ is the unique monic polynomial $\mu_{\alpha}[K](X)$ of least degree in $K[X]$ for which $\alpha$ is a root. We write $\mu_{\alpha}(X)$ for $\mu_{\alpha}[K](X)$ when $K$ is understood. Elements $\alpha, \beta \in L$ are conjugates over $K$ if they have the same minimal polynomial over $K$. The smallest subfield of $L$ containing $K$ and $\alpha$ is denoted by $K(\alpha)$.

The splitting field $K_{f}$ of $f \in K[X]$ is the smallest extension of $K$ containing all the roots of $f$. A finite extension $L / K$ is normal if for all irreducible polynomials $f(X) \in K[X]$, either $f(X)$ splits or has no root in $L$. Any normal extension over $K$ is the splitting field of some polynomial in $K[X]$. An extension $L / K$ is separable if for all irreducible polynomials $f(X) \in K[X]$ there are no multiple roots in $L$. A normal and separable finite extension $L / K$ is a Galois extension.

The Galois group $\operatorname{Gal}(L / K)$ of $L / K$ is the subgroup of automorphisms $\sigma$ of $L$ that leaves $K$ fixed, i.e. $\sigma(\alpha)=\alpha$ for all $\alpha \in K$. The Galois group Gal $(f)$ of 
$f \in K[X]$ is $\operatorname{Gal}\left(K_{f} / K\right)$. For a subgroup $G$ of automorphisms of $L$, the fixed field $L^{G}$ is the largest subfield of $L$ fixed by $G$. We now state the fundamental theorem of Galois.

Theorem 1.1. [5, Theorem 1.1, Chapter VI] Let $L / K$ be a Galois extension with Galois group $G$. There is a one-to-one correspondence between subfields $E$ of $L$ containing $K$ and subgroups $H$ of $G$, given by $E \rightleftharpoons L^{H}$. The Galois group of $\operatorname{Gal}(L / E)$ is $H$ and $E / K$ is a Galois extension if and only if $H$ is a normal subgroup of $G$. If $H$ is a normal subgroup of $G$ and $E=L^{H}$ then $\operatorname{Gal}(E / K)$ is isomorphic to the quotient group $G / H$.

\subsection{Presenting algebraic numbers, number fields and Galois groups}

The algorithms we describe take objects like algebraic numbers, number fields etc. as input. We define sizes of these objects. Integers are encoded in binary. A rational $r$ is given by coprime integers $a, b$ such that $r=a / b$. Thus, size $(r)$ is size $(a)+\operatorname{size}(b)$. A polynomial $T(X)=a_{0}+\ldots+a_{n} X^{n} \in \mathbb{Q}[X]$ is given by a list of its coefficients. Thus, size $(T)$ is defined as $\sum \operatorname{size}\left(a_{i}\right)$.

A number field is a finite extension of $\mathbb{Q}$. Let $K / \mathbb{Q}$ be a number field of degree $n$. By the primitive element theorem [5, Theorem 4.6, Chapter V], there is an algebraic number $\eta \in K$ such that $K=\mathbb{Q}(\eta)$. Such an element is a primitive element of $K / \mathbb{Q}$ and its minimal polynomial is a primitive polynomial. Let $\mu_{\eta}(X)$ be the minimal polynomial of $\eta$ over $\mathbb{Q}$. Then the field $K$ can be written as the quotient $K=\mathbb{Q}[X] / \mu_{\eta}(X)$. Thus $K$ can be presented by giving a primitive polynomial for $K / \mathbb{Q}$. We can assume that $\eta$ is an algebraic integer and hence its minimal polynomial $\mu_{\eta}(X)$ has integer coefficients [5, Proposition 1.1, Chapter VII]. When we say that an algorithm takes a number field $K$ as input we mean that it takes a primitive polynomial $\mu_{\eta}(X)$ for $K$ as input. Thus the input size for $K$, which we denote by size $(K)$, is defined to be size $\left(\mu_{\eta}\right)$.

Suppose $K=\mathbb{Q}(\eta)$ is presented by $\mu_{\eta}(X)$. Notice that each $\alpha \in K$ can be expressed as $\alpha=A_{\alpha}(\eta)$ for a unique polynomial $A_{\alpha}(X) \in \mathbb{Q}[X]$ of degree less than $n$. By size $(\alpha)$ we mean size $\left(A_{\alpha}(X)\right)$. Note that the size of $\alpha \in K$ depends on the primitive element $\eta \in K$. Now, for a polynomial $f(X)=a_{0}+\ldots+a_{m} X^{m}$ in $K[X]$ we define size $(f)$ to be $\sum \operatorname{size}\left(a_{i}\right)$.

Let $f(X) \in \mathbb{Q}[X]$ of degree $n$. For an algorithm purporting to compute $\operatorname{Gal}(f)$, one possibility is that it outputs the complete multiplication table for Gal $(f)$. However, this could be exponential in size $(f)$ as $\operatorname{Gal}(f)$ can be as large as $n$ !. A succinct presentation of $\operatorname{Gal}(f)$ is as a permutation group acting on the roots of $f$ since elements of $\operatorname{Gal}(f)$ permute the roots of $f$ and are completely determined by their action on the roots of $f$. Thus, by numbering the roots of $f$, we can consider Gal $(f)$ as a subgroup of the symmetric group $S_{n}$ (note here that $\mathrm{Gal}(f)$ is determined only up to conjugacy as the numbering of the roots is arbitrary). Since any subgroup of $S_{n}$ has a generator set of size $n-1$ (see e.g. [8]), we can present Gal $(f)$ in size polynomial in $n$. Thus, by

computing Gal $(f)$ we mean finding a small generator set for it as a subgroup of $S_{n}$. Determining Gal $(f)$ as a subgroup of $S_{n}$ is a reasonable way of describing 
the output. Algorithmically, we can answer several natural questions about a subgroup $G$ of $S_{n}$ given by generator set in polynomial time. E.g. testing if $G$ is solvable, finding a composition series for $G$ etc. [8].

Previous complexity results

As mentioned, the best known algorithm for computing the Galois group of a polynomial is due to Landau [3].

Theorem 1.2 (Landau). There is a deterministic algorithm that takes as input a number field $K$, a polynomial $f(X) \in K[X]$ and a positive integer $b$ in unary, and in time bounded by size $(f)$, size $(K)$ and $b$, decides if $\mathrm{Gal}\left(K_{f} / K\right)$ has at most $b$ elements, and if so computes $\mathrm{Gal}\left(K_{f} / K\right)$ by finding the entire multiplication table of $\mathrm{Gal}\left(K_{f} / K\right)$ (and hence also by giving the generating set of $\mathrm{Gal}\left(K_{f} / K\right)$ as a permutation group on the roots of $\left.f(X)\right)$.

The algorithm first computes a primitive element $\theta$ of $K_{f}$. Determining $\mathrm{Gal}(f)$ amounts to finding the action of the automorphisms on $\theta$. Subsequently, Landau and Miller [4 gave their polynomial-time solvability test.

Theorem 1.3 (Landau-Miller). Given $f(X) \in \mathbb{Q}[X]$ there is a deterministic polynomial-time algorithm for testing if $\mathrm{Gal}(f)$ is solvable.

\section{Preliminaries}

We recall some permutation group theory from Wielandt's book 9 . Let $\Omega$ be a finite set. The symmetric group $\operatorname{Sym}(\Omega)$ is the group of all permutations on $\Omega$. By a permutation group on $\Omega$ we mean a subgroup of $\operatorname{Sym}(\Omega)$. For $\alpha \in \Omega$ and $g \in \operatorname{Sym}(\Omega)$, let $\alpha^{g}$ denote the image of $\alpha$ under the permutation $g$. For $A \subseteq \operatorname{Sym}(\Omega), \alpha^{A}$ denotes the set $\left\{\alpha^{g}: g \in A\right\}$. In particular, for $G \leq \operatorname{Sym}(\Omega)$ the $G$-orbit containing $\alpha$ is $\alpha^{G}$. The $G$-orbits form a partition of $\Omega$. Given $G \leq \operatorname{Sym}(\Omega)$ by a generating set $A$ and $\alpha \in \Omega$, there is a polynomial-time algorithm to compute $\alpha^{G} 8$.

For $\Delta \subseteq \Omega$ and $g \in \operatorname{Sym}(\Omega), \Delta^{g}$ denotes $\left\{\alpha^{g}: \alpha \in \Delta\right\}$. The setwise stabilizer of $\Delta$, i.e. $\left\{g \in G: \Delta^{g}=\Delta\right\}$, is denoted by $G_{\Delta}$. If $\Delta$ is the singleton set $\{\alpha\}$ we write $G_{\alpha}$ instead of $G_{\{\alpha\}}$. For any $\Delta$ by $\left.G\right|_{\Delta}$ we mean $G_{\Delta}$ restricted to $\Delta$. An often used result is the orbit-stabilizer formula stated below [9, Theorem 3.2].

Theorem 2.1 (Orbit-stabilizer formula). Let $G$ be a permutation group on $\operatorname{Sym}(\Omega)$ and let $\alpha$ be any element of $\Omega$ then the order of the group $G$ is given by $\# G=\# G_{\alpha} . \# \alpha^{G}$.

A permutation group $G$ on $\Omega$ is transitive if there is a single $G$-orbit. Suppose $G \leq \operatorname{Sym}(\Omega)$ is transitive. Then a non-empty subset $\Delta$ of $\Omega$ is a $G$-block if for all $g \in G$ either $\Delta^{g}=\Delta$ or $\Delta^{g} \cap \Delta=\emptyset$. For every $G, \Omega$ is a block and each singleton $\{\alpha\}$ is a block. These are the trivial blocks of $G$. A transitive group $G$ is primitive if it has only trivial blocks and it is imprimitive if it has nontrivial 
blocks. A $G$-block $\Delta$ is a maximal subblock of a $G$-block $\Sigma$ if $\Delta \subset \Sigma$ and there is no $G$-block $\Upsilon$ such that $\Delta \subset \Upsilon \subset \Omega$. Let $\Delta$ and $\Sigma$ be two $G$-blocks. A chain $\Delta=\Delta_{0} \subset \ldots \subset \Delta_{t}=\Sigma$ is a maximal chain of $G$-blocks between $\Delta$ and $\Sigma$ if for all $i, \Delta_{i}$ is a maximal subblock of $\Delta_{i+1}$.

For a $G$-block $\Delta$ and $g \in G, \Delta^{g}$ is also a $G$-block such that $\# \Delta=\# \Delta^{g}$. Let $\Delta$ and $\Sigma$ be two $G$-blocks such that $\Delta \subseteq \Sigma$. The $\Delta$-block system of $\Sigma$, is the collection

$$
\mathcal{B}(\Sigma / \Delta)=\left\{\Delta^{g}: g \in G \text { and } \Delta^{g} \subseteq \Sigma\right\} .
$$

The set $\mathcal{B}(\Sigma / \Delta)$ is a partition of $\Sigma$. It follows that $\# \Delta$ divides $\# \Sigma$ and by index of $\Delta$ in $\Sigma$, which we denote by $[\Sigma: \Delta]$, we mean $\# \mathcal{B}(\Sigma / \Delta)=\frac{\# \Sigma}{\# \Delta}$. We will use $\mathcal{B}(\Delta)$ to denote $\mathcal{B}(\Omega / \Delta)$. We state the connection between blocks and subgroups [9, Theorem 7.5].

Theorem 2.2 (Galois correspondence of blocks). Let $G \leq \operatorname{Sym}(\Omega)$ be transitive and $\alpha \in \Omega$. For $G \geq H \geq G_{\alpha}$ the orbit $\Delta=\alpha^{H}$ is a $G$-block and $G_{\Delta}=H$. The correspondence $\alpha^{H}=\Delta \rightleftharpoons G_{\Delta}=H$ is a one-to-one correspondence between $G$-blocks $\Delta$ containing $\alpha$ and subgroups $H$ of $G$ containing $G_{\alpha}$. Furthermore for $G$-blocks $\Delta \subseteq \Sigma$ we have $\left[G_{\Sigma}: G_{\Delta}\right]=[\Sigma: \Delta]$.

Let $G \leq \operatorname{Sym}(\Omega)$ be transitive and $\Delta$ and $\Sigma$ be two $G$-blocks such that $\Delta \subseteq \Sigma$. Let $G(\Sigma / \Delta)$ denote the group $\left\{g \in G: \Upsilon^{g}=\Upsilon\right.$ for all $\left.\Upsilon \in \mathcal{B}(\Sigma / \Delta)\right\}$. We write $G^{\Delta}$ for the group $\mathrm{G}(\Omega / \Delta)$. For any $g \in G_{\Sigma}$, since $g$ setwise stabilises $\Sigma, g$ permutes the elements of $\mathcal{B}(\Sigma / \Delta)$. Hence for any $\Upsilon \in \mathcal{B}(\Sigma / \Delta)$ we have $\Upsilon^{g^{-1} \mathrm{G}(\Sigma / \Delta) g}=\Upsilon$. Thus, $\mathrm{G}(\Sigma / \Delta)$ is a normal subgroup of $G_{\Sigma}$. In particular, $G^{\Delta}$ is a normal subgroup of $G$.

Remark. The following two lemmata are quite standard in permutation group theory. For the reader's convenience we have included short proofs. The following lemma lists important properties of $G^{\Delta}$.

\section{Lemma 2.3.}

1. For a $G$-block $\Delta \subseteq \Sigma, \mathrm{G}(\Sigma / \Delta)$ is the largest normal subgroup of $G_{\Sigma}$ contained in $G_{\Delta}$.

2. Let $\Sigma$ be $G$-block then $\left.G^{\Sigma} \hookrightarrow \prod_{\Upsilon \in \mathcal{B}(\Sigma)} G\right|_{\Upsilon}$.

3. Let $\Delta$ be a G-subblock of $\Sigma$ then $\frac{G_{\Sigma}}{\mathrm{G}(\Sigma / \Delta)}$ is a faithful permutation group on $\mathcal{B}(\Sigma / \Delta)$ and is primitive when $\Delta$ is a maximal subblock.

4. The quotient group $G^{\Sigma} / G^{\Delta}$ can be embedded as a subgroup of $\left(\frac{G_{\Sigma}}{\mathrm{G}(\Sigma / \Delta)}\right)^{l}$ for somel.

Proof. Let $N \subseteq G_{\Delta}$ be a normal subgroup of $G_{\Sigma}$. Since $\Delta^{N}=\Delta$, and since $G_{\Sigma}$ acts transitively on $\mathcal{B}(\Sigma / \Delta)$, for any $\Upsilon \in \mathcal{B}(\Sigma / \Delta)$ there is a $g \in G_{\Sigma}$ such that $\Upsilon=\Delta^{g}$. Therefore, $\Upsilon^{N}=\Delta^{g N}=\Delta^{N g}=\Upsilon$ for each $\Upsilon \in \mathcal{B}(\Sigma / \Delta)$. Thus $N \subseteq \mathrm{G}(\Sigma / \Delta)$. Since $\mathrm{G}(\Sigma / \Delta) \unlhd G_{\Sigma}$ we have proved part 1 . 
Part 2 directly follows from the definition of $G^{\Sigma}$. Part 3 follows from the fact that $g, h \in G_{\Sigma}$ have the same action on $\mathcal{B}(\Sigma / \Delta)$ precisely when $g \mathrm{G}(\Sigma / \Delta)=$ $h \mathrm{G}(\Sigma / \Delta)$. The nontrivial $\frac{G_{\Sigma}}{\mathrm{G}(\Sigma / \Delta)}$-blocks of $\mathcal{B}(\Sigma / \Delta)$ are in 1-1 correspondence with the $G$-blocks properly between $\Delta$ and $\Sigma$. Thus, $\frac{G_{\Sigma}}{\mathrm{G}(\Sigma / \Delta)}$ is primitive if and only if $\Delta$ is a maximal subblock of $\Sigma$.

For Part 4 notice that we have the group isomorphism

$$
\frac{\left.G\right|_{\Upsilon}}{\left.\mathrm{G}\left(\Upsilon / \Delta_{\Upsilon}\right)\right|_{\Upsilon}} \cong \frac{G_{\Upsilon}}{\mathrm{G}\left(\Upsilon / \Delta_{\Upsilon}\right)}
$$

for each $\Upsilon \in \mathcal{B}(\Sigma)$. As $G^{\Delta}=\left.G^{\Sigma} \cap \prod \mathrm{G}\left(\Upsilon / \Delta_{\Upsilon}\right)\right|_{\Upsilon}$ we have

$$
G^{\Sigma} / G^{\Delta} \hookrightarrow \prod_{\Upsilon \in \mathcal{B}(\Sigma)} \frac{\left.G\right|_{\Upsilon}}{\left.\mathrm{G}\left(\Upsilon / \Delta_{\Upsilon}\right)\right|_{\Upsilon}}=\prod_{\Upsilon \in \mathcal{B}(\Sigma)} \frac{G_{\Upsilon}}{\mathrm{G}\left(\Upsilon / \Delta_{\Upsilon}\right)}
$$

Let $g \in G$ such that $\Delta^{g}=\Delta_{\Upsilon}$. Then, $G_{\Upsilon}=g^{-1} G_{\Sigma} g$ and $\mathrm{G}\left(\Upsilon / \Delta_{\Upsilon}\right)=$ $g^{-1} \mathrm{G}(\Sigma / \Delta) g$. Thus, $\frac{G_{\Sigma}}{\mathrm{G}(\Sigma / \Delta)}$ and $\frac{G_{\Upsilon}}{\mathrm{G}\left(\Upsilon / \Delta_{\Upsilon}\right)}$ are isomorphic, which implies that $G^{\Sigma} / G^{\Delta}$ is isomorphic to a subgroup of $\left(\frac{G_{\Sigma}}{\mathrm{G}(\Sigma / \Delta)}\right)^{l}$ for some $l$.

Lemma 2.4. Let $G \leq \operatorname{Sym}(\Omega)$ be transitive and $N \unlhd G$. Let $\alpha \in \Omega$. Then the $N$-orbit $\alpha^{N}$ is a $G$-block and the collection of $N$-orbits is an $\alpha^{N}$-block system of $\Omega$ under $G$ action. If $N \neq\{1\}$ then $\left\|\alpha^{N}\right\|>1$. Furthermore, if $G_{\alpha} \leq N \neq G$ then the $\alpha^{N}$-block system is nontrivial implying that $G$ is not primitive.

Proof. Let $\alpha \in \Omega$ and $g \in G$. Then $\left(\alpha^{N}\right)^{g}=\alpha^{N g}=\alpha^{g N}=\left(\alpha^{g}\right)^{N}$. Thus $\left(\alpha^{N}\right)^{g}$ and $\alpha^{N}$ are $N$-orbits, and hence are identical or disjoint. Thus, $\alpha^{N}$ is a $G$-block and the $N$-orbits form a block system. Clearly, if $\alpha^{N}=\{\alpha\}$ then $N=\{1\}$. Finally, by the Orbit-Stabilizer formula $\# G=\# \Omega \cdot \# G_{\alpha}$ and $\# N=\# \alpha^{N} \cdot \# G_{\alpha}$. Thus, if $\{1\} \neq N \neq G$ then $\alpha^{N}$ is a proper $G$-block.

\section{$3 \quad$ Nilpotence testing for Galois groups}

First we recall crucial properties of nilpotent transitive permutation groups. These are standard group theoretic facts that we assemble together and, for the sake of completeness, provide proof sketches where necessary. We start with a characterization of finite nilpotent groups. Let $G$ be a finite group and $p_{1}, \ldots, p_{k}$ be the prime factors of $\# G$. For each $i$, let $G_{p_{i}}$ be a $p_{i}$-Sylow subgroup of $G$. Then $G$ is nilpotent if and only if $G$ is the (internal) direct product $G_{p_{1}} \times \ldots \times G_{p_{k}}$. Consequently, $G_{p_{i}}$ is the unique $p_{i}$-Sylow subgroup of $G$ for each $i$ and hence $G_{p_{i}} \triangleleft G$.

Lemma 3.1. Let $G \leq \operatorname{Sym}(\Omega)$ be transitive and nilpotent, and $p$ be any prime. Then

(1) The prime $p$ divides $\# G$ if and only if $p$ divides $\# \Omega$. 
(2) If $p \mid \# G$ and $\alpha \in \Omega$ then there is a block $\Sigma_{p}^{\alpha}$ containing $\alpha$ such that $\# \Sigma_{p}^{\alpha}$ is the highest power of $p$ that divides $\# \Omega$.

(3) Let $\Delta$ be any $G$-block containing $\alpha$ such that $\# \Delta=p^{l}$ and suppose $p$ divides $\# G$. Then $\Delta \subseteq \Sigma_{p}^{\alpha}$. Also, for $q \neq p$, the $q$-Sylow subgroup of $G_{\Delta}$ is given by $G_{q} \cap G_{\Delta}=G_{q} \cap G_{\alpha}$.

Proof. Part (1): As $G$ is transitive, $\# \Omega$ divides $\# G$. Hence, each prime factor of $\# \Omega$ divides $\# G$. Let $p$ be a prime factor of $\# G$. For $\alpha \in \Omega$, let $\Sigma_{p}^{\alpha}=\alpha^{G_{p}}$. Since $G_{p}$ is transitive on $\Sigma_{p}^{\alpha}$, it follows from the Orbit-Stabilizer formula that $\# \Sigma_{p}^{\alpha}$ divides $\# G_{p}$. Hence $\# \Sigma_{p}^{\alpha}$ is $p^{l}$ for some $l$. Since $G_{p} \triangleleft G$, by Lemma 2.4 it follows that its orbit $\Sigma_{p}^{\alpha}$ is $G$-block which contains more than one element of $\Omega$. Hence $\# \Sigma_{p}^{\alpha}=p^{l}$ for some $l>0$. Since $p$ divides the cardinality of a $G$-block $\Sigma_{p}^{\alpha}, p$ divides $\# \Omega$.

Part (2): From the Galois correspondence of $G$-blocks (Theorem 2.2) we have $\left[\Omega: \Sigma_{p}^{\alpha}\right]=\left[G: G_{\Sigma_{p}^{\alpha}}\right]$. Notice that $p$ is not a factor of $\left[G: G_{p}\right]$ as $G_{p}$ is the $p$-Sylow subgroup of $G$. Since $G_{p} \triangleleft G_{\Sigma_{p}^{\alpha}}$ it follows that $p$ is not a factor of $\left[G: G_{\Sigma_{p}^{\alpha}}\right]$. Hence $p$ is not a factor of $\left[\Omega: \Sigma_{p}^{\alpha}\right]$.

Part (3): notice that $G_{\Delta}$ is a nilpotent group with the unique normal $q$ Sylow subgroup $G_{q} \cap G_{\Delta}$. Thus, $G_{\Delta}=\prod_{q}\left(G_{q} \cap G_{\Delta}\right)$. By Theorem 2.2 we have

$$
\# \Delta=\left[G_{\Delta}: G_{\alpha}\right]=\prod_{q}\left[G_{q} \cap G_{\Delta}: G_{q} \cap G_{\alpha}\right] .
$$

Since $G_{q} \cap G_{\Delta}$ is a $q$-group, $p$ divides $\left[G_{q} \cap G_{\Delta}: G_{q} \cap G_{\alpha}\right]$ if and only if $q=p$. However, in Equation 1. \# $\Delta$ is a power of $p$. This forces $\left[G_{q} \cap G_{\Delta}: G_{q} \cap G_{\alpha}\right]=1$ for all $q \neq p$. Thus $G_{q} \cap G_{\Delta}=G_{q} \cap G_{\alpha}$ for $q \neq p$. Therefore, $G_{\Delta}$ is the product group $G_{p} \cap G_{\Delta} \times \prod_{q \neq p} G_{q} \cap G_{\alpha}$. Since $G_{\Sigma_{p}^{\alpha}}$ contains both $G_{p}$ and $G_{\alpha}$ we have $G_{\Sigma_{p}^{\alpha}} \geq G_{\Delta}$. Thus, $\Delta$ is a $G$-subblock of $\Sigma_{p}^{\alpha}$. $1.1])$.

We recall a result about permutation $p$-groups (see e.g. Luks [7, Lemma

Lemma 3.2. Let $G \leq \operatorname{Sym}(\Omega)$ be a transitive p-group and $\Delta$ be a maximal $G$-block. Then $[\Omega: \Delta]=p$ and $G_{\Delta}=\mathrm{G}(\Omega / \Delta)=G^{\Delta}$ is a normal group of index $p$ in $G$.

The next lemma is an easy consequence of Lemma 3.2 and it states a useful property of permutation $p$-groups.

Lemma 3.3. Let $H \leq \operatorname{Sym}(\Omega)$ be a transitive $p$-group and $\alpha \in \Omega$. Let $\{\alpha\}=$ $\Delta_{0} \subset \ldots \subset \Delta_{t}=\Omega$ be any maximal chain of $H$-blocks. Then

1. $\left[\Delta_{i+1}: \Delta_{i}\right]=p$ for all $0 \leq i<t$.

2. $H\left(\Delta_{i+1} / \Delta_{i}\right)=H_{\Delta_{i}}$. Hence, $H_{\Delta_{i}} \triangleleft H_{\Delta_{i+1}}$ and the quotient $H_{\Delta_{i+1}} / H_{\Delta_{i}}$ is cyclic of order $p$. 
We continue with the notation of Lemma 3.1. In the next lemma we show that the block structure of transitive nilpotent permutation group $G$ is similar to the block structure $p$-groups.

Lemma 3.4. Let $G$ be a nilpotent transitive permutation group on $\Omega$ and let $p$ be a prime factor of $\# G$. Let $\Delta$ be any subset of $\Sigma_{p}^{\alpha}$. Then $\Delta$ is a G-block if and only if $\Delta$ is a $G_{p}$ block (in its transitive action on $\Sigma_{p}^{\alpha}$ ).

Proof. Let $H$ denote the $p$-Sylow subgroup $G_{p}$. Let $\widehat{H}$ denote the product $\prod_{q \neq p} G_{q}$ of all other Sylow subgroups of $G$. Then $G=H \times \widehat{H}$. Recall that $\Sigma_{p}^{\alpha}$ is the $H$-orbit of $\alpha$.

Firstly any $G$-block $\Delta \subseteq \Sigma_{p}^{\alpha}$ is an $H$-block. To prove the converse consider any $H$-block $\Sigma \subseteq \Sigma_{p}^{\alpha}$. Consider the group $G^{\prime}=H_{\Sigma} \times\left(\widehat{H} \cap G_{\alpha}\right)$. Firstly notice that the group $G^{\prime}$ is a subgroup of $G_{\Sigma_{p}^{\alpha}}$. Also since $G_{\alpha}$ is nilpotent, we have $G_{\alpha}=H_{\alpha} \times\left(\widehat{H} \cap G_{\alpha}\right)$. Furthermore since $\Sigma$ is a $H$-block, we have $H_{\Sigma} \geq H_{\alpha}$. Therefore $G^{\prime} \geq G_{\alpha}$ and by the Galois correspondence of blocks (Theorem 2.2), $\Sigma=\alpha^{G^{\prime}}$ is a $G$-block and $G_{\Sigma}=G^{\prime}$.

We give a characterisation of nilpotent transitive permutation groups by properties of maximal chains of $G$-blocks between $\{\alpha\}$ and $\Sigma_{p}^{\alpha}$ which is crucial for our polynomial-time nilpotence test. This characterization is probably wellknown to group theorists. However, as we haven't seen it anywhere, we include a proof.

Theorem 3.5. Let $G \leq \operatorname{Sym}(\Omega)$ be a transitive permutation group satisfying properties (1) and (2) of Lemma 3.1 (which are necessary conditions for nilpotence of $G$ ). Fix an $\alpha \in \Omega$. The following statements are equivalent.

(1) $G$ is nilpotent.

(2) For each prime factor $p$ of $\# G$, every maximal chain of $G$-blocks $\{\alpha\}=$ $\Delta_{0} \subset \ldots \subset \Delta_{m}=\Sigma_{p}^{\alpha}$ has the property that $\left[\Delta_{i+1}: \Delta_{i}\right]=p, G_{\Delta_{i}}$ is a normal subgroup of $G_{\Delta_{i+1}}$, and $p$ does not divide the order of $G / G^{\Delta_{m}}$.

(3) For each prime $p$ dividing $\# G$, there is a maximal chain of $G$-blocks $\{\alpha\}=$ $\Delta_{0} \subset \ldots \subset \Delta_{m}=\Sigma_{p}^{\alpha}$ with the property that $\left[\Delta_{i+1}: \Delta_{i}\right]=p, G_{\Delta_{i}}$ is a normal subgroup of $G_{\Delta_{i+1}}$, and $p$ does not divide the order of $G / G^{\Delta_{m}}$.

Proof. Clearly (2) implies (3). It suffices to show that (3) implies (1) and (1) implies (2).

To see that (3) implies (1) it is enough to show that each Sylow subgroup of $G$ is normal. To this end, let $p$ be a prime factor of $\# G$ and let $\{\alpha\}=$ $\Delta_{0} \subset \ldots \subset \Delta_{m}=\Sigma_{p}^{\alpha}$ be a maximal chain of $G$-blocks having the properties mentioned in (3).

Firstly, since $\mathrm{G}\left(\Delta_{i+1} / \Delta_{i}\right)$ is the largest normal subgroup of $G_{\Delta_{i+1}}$ that is contained in $G_{\Delta_{i}}$ (part 1 of Lemma 2.3), (3) implies that $G_{\Delta_{i}}=\mathrm{G}\left(\Delta_{i+1} / \Delta_{i}\right)$. Furthermore it follows from Lemma 2.3 that there is a positive integer $l_{i}$ for each 
$i$ such that the quotient group $G^{\Delta_{i+1}} / G^{\Delta_{i}}$ is embeddable in an $l_{i}$-fold product of copies of $\frac{G_{\Delta_{i+1}}}{\mathrm{G}\left(\Delta_{i+1} / \Delta_{i}\right)}=G_{\Delta_{i+1}} / G_{\Delta_{i}}$. Since $\left[G_{\Delta_{i+1}}: G_{\Delta_{i}}\right]=p$ it follows that $G^{\Delta_{i+1}} / G^{\Delta_{i}}$ is a $p$-group for each $i$. As $\# G^{\Delta_{m}}=\prod_{i=0}^{m-1}\left[G^{\Delta_{i+1}}: G^{\Delta_{i}}\right], G^{\Delta_{m}}$ is also a $p$-group. Since $G^{\Delta_{m}} \triangleleft G$ and $p$ does not divide $\left[G: G^{\Delta_{m}}\right]$ it follows that $G^{\Delta_{m}}$ is a normal $p$-Sylow subgroup of $G$. The nilpotence of $G$ follows as this holds for all prime factors of $\# G$.

Next, we show that (1) implies (2). Suppose $G$ is nilpotent. Let $p$ be a prime factor of \#G and $\alpha \in \Omega$. Let $H$ be the $p$-Sylow subgroup $G_{p}$ of $G$ and let $\widehat{H}=\prod_{q \neq p} G_{q}$ be the product of all its other Sylow subgroups. Let $\{\alpha\}=\Delta_{0} \subset$ $\Delta_{1} \subset \ldots \subset \Delta_{m}=\Sigma_{p}^{\alpha}$ be any maximal chain of $G$-blocks between $\alpha$ and $\Sigma_{p}^{\alpha}$. It follows from Lemma 3.4 that the chain $\{\Delta\}_{0 \leq i \leq m}$ is a maximal chain of $G_{p^{-}}$ blocks. By Lemma 3.3 we have $\left[\Delta_{i+1}: \Delta_{i}\right]=p, H_{\Delta_{i}} \triangleleft H_{\Delta_{i+1}}$, and $H_{\Delta_{i+1}} / H_{\Delta_{i}}$ is cyclic of order $p$. The group $G_{\Delta_{i}}=H_{\Delta_{i}} \times \widehat{H}_{\Delta_{i}}$ and $G_{\Delta_{i+1}}=H_{\Delta_{i+1}} \times \widehat{H}_{\Delta_{i+1}}$. Also since $\widehat{H}_{\Delta_{i}}$ is the product of $q$-Sylow subgroups of $H_{\Delta_{i}}$ where $q$ varies over all prime factors of $\# G$ different from $p$, it follows from Lemma 3.1 that $\widehat{H}_{\Delta_{i}}=\widehat{H}_{\alpha}$. Therefore $G_{\Delta_{i}} \triangleleft G_{\Delta_{i+1}}$ and quotient group $G_{\Delta_{i+1}} / G_{\Delta_{i}} \cong H_{\Delta_{i+1}} / H_{\Delta_{i}}$. The group $G / G^{\Delta_{m}}$ acts faithfully on $\mathcal{B}\left(\Omega / \Delta_{m}\right)$ and is transitive under this action. Since $p \nmid\left[\Omega: \Delta_{m}\right], p$ cannot divide the order of $G / G^{\Delta_{m}}$ (Lemma 3.1).

The following lemma is crucial for the nilpotence testing algorithm. If $G$ is nilpotent then, for each prime factor $p$ of $\# G$, the lemma implies that no matter how the maximal chain of blocks $\Delta_{i}$ of Theorem 3.5 is constructed, it must terminate in $\Sigma_{p}^{\alpha}$.

Lemma 3.6. Let $G$ be a transitive nilpotent permutation group on $\Omega$. Let $p$ be any prime dividing $\# G$. Let $\Delta$ be any $G$-block such that $\# \Delta=p^{l}$ for some integer $l \geq 0$. Let $m$ be the highest power of $p$ that divides $\# \Omega$. If $l<m$ then we have

1. There exists a $G$-block $\Sigma$ such that $\Delta$ is a maximal $G$-subblock of $\Sigma$ and $[\Sigma: \Delta]=p$.

2. For all $G$-blocks $\Sigma$ such that $\Delta$ is a maximal $G$-subblock of $\Sigma$ and $[\Sigma$ : $\Delta]=p, G_{\Delta}$ is a normal subgroup of $G_{\Sigma}$.

Proof. Since $\# \Delta$ is $p^{l}$ it follows that $\Delta$ is a $G$-subblock of $\Sigma_{p}^{\alpha}$ (Lemma 3.1). It follows from Lemma 3.4 that $\Delta$ is a $G_{p}$-block on the transitive action of $G_{p}$ on $\Sigma_{p}^{\alpha}$. Furthermore if $l<m$ there is a $G_{p}$-block $\Sigma$ (and hence by Lemma 3.4 a $G$-block) such that $\Sigma_{p}^{\alpha} \supseteq \Sigma \supset \Delta$ and $[\Sigma: \Delta]=p$. This proves part 1 .

Let $\alpha \in \Delta$. It follows from Lemma 3.1 that for $q \neq p$ the $q$-Sylow subgroup of $G_{\Sigma}$ and $G_{\Delta}$ are both $G_{q} \cap G_{\alpha}$. Let $G_{p}$ be $\prod_{q \neq p} G_{q}$. The groups $G_{\Sigma}$ and $G_{\Delta}$ are $\left(G_{p} \cap G_{\Sigma}\right) \times\left(\widehat{G}_{p} \cap G_{\alpha}\right)$ and $\left(G_{p} \cap G_{\Delta}\right) \times\left(\widehat{G}_{p} \cap G_{\alpha}\right)$ respectively. Moreover, $G_{p} \cap G_{\Sigma}$ and $G_{p} \cap G_{\Delta}$ are $p$-groups with index $\left[G_{p} \cap G_{\Sigma}: G_{p} \cap G_{\Delta}\right]=\left[G_{\Sigma}\right.$ : $\left.G_{\Delta}\right]=[\Sigma: \Delta]=p$. Therefore, $G_{p} \cap G_{\Delta}$ is normal in $G_{p} \cap G_{\Sigma}$. Thus, $G_{\Delta}=$ $\left(G_{p} \cap G_{\Delta}\right) \times\left(\widehat{G}_{p} \cap G_{\alpha}\right)$ is normal in $G_{\Sigma}=\left(G_{p} \cap G_{\Sigma}\right) \times\left(\widehat{G}_{p} \cap G_{\alpha}\right)$ and $\frac{G_{\Sigma}}{G_{\Delta}}=\frac{G_{p} \cap G_{\Sigma}}{G_{p} \cap G_{\Delta}}$ is isomorphic to $\mathbb{Z}_{p}$. 


\subsection{The nilpotence test}

Given $f(X) \in \mathbb{Q}[X]$ our goal is to test if $\mathrm{Gal}(f)$ is nilpotent. We can assume that $f(X)$ is irreducible. For, otherwise we can compute the irreducible factors of $f(X)$ over $\mathbb{Q}$ using the LLL algorithm, and perform the nilpotence test on each distinct irreducible factor. This suffices because nilpotent groups are closed under products and subgroups. Let $G$ be $\operatorname{Gal}(f)$. We consider $G$ as a subgroup of $\operatorname{Sym}(\Omega)$, where $\Omega$ is the set of roots of $f(X)$. Since $f$ is irreducible, $G$ is transitive on $\Omega$.

For any $G$-block $\Delta$, let $\mathbb{Q}_{\Delta}$ be the fixed field of the splitting field $\mathbb{Q}_{f}$ under the automorphisms of $G_{\Delta}$. Let $\Delta$ be a $G$-block containing $\alpha$. Since $G_{\Delta} \geq G_{\alpha}$, $\mathbb{Q}_{\Delta}$ is a subfield of $\mathbb{Q}_{\{\alpha\}}=\mathbb{Q}(\alpha)$.

We describe the main idea. By Theorem 3.5 $G$ is nilpotent if and only if for all primes $p$ that divide the order of $G$, there is a maximal chain of $G$-blocks $\{\alpha\}=\Delta_{0} \subset \ldots \subset \Delta_{m}$ satisfying conditions of part (3) of Theorem 3.5. We show these conditions can be verified in polynomial time once the tower of fields $\mathbb{Q}(\alpha)=\mathbb{Q}_{\Delta_{0}} \supset \ldots \supset \mathbb{Q}_{\Delta_{m}}$ are known. Thus, for testing nilpotence of $G$ we will first need a polynomial-time algorithm that computes $\mathbb{Q}_{\Delta_{i}}$. The following theorem is essentially due to Landau and Miller [4] restated in a form suitable for our application.

Theorem 3.7. Let $f(X) \in \mathbb{Q}[X]$ be irreducible, $G=\mathrm{Gal}(f)$ be its Galois group and $\Omega$ be the set of roots of $f$. Let $\Delta \subseteq \Omega$ be any $G$-block and $\alpha \in \Delta$. There is an algorithm that given a primitive polynomial $\mu_{\Delta}(X) \in \mathbb{Q}[X]$ of $\mathbb{Q}_{\Delta}$, runs in time polynomial in size $(f)$ and size $\left(\mu_{\Delta}\right)$ and computes a primitive polynomial $\mu_{\Sigma}(X) \in \mathbb{Q}[X]$ of $\mathbb{Q}_{\Sigma}$ for all $G$-blocks $\Sigma$ such that $\Delta$ is a maximal block of $\Sigma$. Moreover size $\left(\mu_{\Sigma}\right)$ is at most a polynomial in size $(f)$ and is independent of $\operatorname{size}\left(\mu_{\Delta}\right)$.

We now give the algorithm for testing nilpotence.

We prove that Algorithm 1 runs in polynomial time. For the steps 1 and 5 note that for polynomials $f$ with solvable Galois groups, as a byproduct of the Landau-Miller test [4], the prime factors of $\# \mathrm{Gal}(f)$ can be found in polynomial time (see also Theorem 4.3). We explain how step 3 can be done in polynomial time using Theorem 3.7. We construct $\mathbb{Q}_{\Delta_{i}}$ inductively starting with $\mathbb{Q}_{\Delta_{0}}=$ $\mathbb{Q}(\alpha)$. Assume we have computed $\mathbb{Q}_{\Delta_{i}}$. Using Theorem 3.7 we compute $\mathbb{Q}_{\Sigma}$ for each $G$-block $\Sigma$ containing $\Delta_{i}$ as a maximal $G$-subblock. Among them choose a $\mathbb{Q}_{\Sigma}$ for which $\left[\Sigma: \Delta_{i}\right]=p$ and let $\mathbb{Q}_{\Delta_{i+1}}$ be $\mathbb{Q}_{\Sigma}$. The inductive construction of $\mathbb{Q}_{\Delta_{i+1}}$ from $\mathbb{Q}_{\Delta_{i}}$ can be done in time bounded by a polynomial in size $(f)$. Putting it together we have the following proposition.

Proposition 3.8. Algorithm 1 runs in time polynomial in size $(f)$.

We now argue its correctness. Part (1) of Theorem 3.5 implies that if $G$ is nilpotent then Algorithm 1 accepts. Conversely, suppose the algorithm accepts. Then for each prime $p$ dividing $\# G$ we have a maximal chain of $G$-blocks $\{\alpha\}=$ $\Delta_{0} \subset \ldots \subset \Delta_{m}$ such that $\mathbb{Q}_{\Delta_{i}} / \mathbb{Q}_{\Delta_{i+1}}$ are normal extensions for each $0 \leq i<m$ (this we verify in step 4 of Algorithm 1 . Recall that $\mathbb{Q}_{\Delta_{i}}$ is the fixed field of 
Input: A polynomial $f(X) \in \mathbb{Q}[X]$ of degree $n$

Output: "Accept" if Gal $(f)$ is nilpotent; "Reject" otherwise

Verify that $f(X)$ is solvable;

1 Compute the set $P$ of all the prime factors of \#Gal $(f)$;

Let $G \leq \operatorname{Sym}(\Omega)$ denote the Galois group of $f$, where $\Omega$ is the set of roots of $f$.

2 for every $p \in P$ do

if $p$ does not divide $n$ then

print Reject

end

Let $m$ be the highest power of $p$ dividing $n$.

$3 \quad$ Attempt to compute the tower $\mathbb{Q}_{\Delta_{m}} \subset \ldots \subset \mathbb{Q}_{\Delta_{0}}$ for a maximal chain of $G$-blocks $\{\alpha\}=\Delta_{0} \subset \ldots \subset \Delta_{m}$ such that $\left[\mathbb{Q}_{\Delta_{i+1}}: \mathbb{Q}_{\Delta_{i}}\right]=p$.

4 if Step 3 fails or $\mathbb{Q}_{\Delta_{i+1}}$ is not normal over $\mathbb{Q}_{\Delta_{i}}$ then

print Reject

end

Let $\mu_{\Delta_{m}}(X)$ be the primitive polynomial for $\mathbb{Q}_{\Delta_{m}}$

$\mathbf{5} \quad$ if $p$ divides \#Gal $\left(\mu_{\Delta_{m}}\right)$ then print Reject

end

end

print Accept

Algorithm 1: Nilpotence test

$\mathbb{Q}_{f}$ w.r.t. $G_{\Delta_{i}}$. Hence by checking $\mathbb{Q}_{\Delta_{i}} / \mathbb{Q}_{\Delta_{i+1}}$ is a normal extension we have verified that $G_{\Delta_{i}} \triangleleft G_{\Delta_{i+1}}$. Also, the splitting field of the primitive polynomial $\mu_{\Delta_{m}}(X)$ is the normal closure of $\mathbb{Q}_{\Delta_{m}}$ over $\mathbb{Q}$. It follows from Lemma 2.3 and Theorem 1.1 that $\operatorname{Gal}\left(\mu_{\Delta_{m}}\right)$ is $G^{\Delta_{m}}$. Hence, by checking $p$ does not divide \#Gal $\left(\mu_{\Delta}\right)$ we have verified that $p$ does not divide \#G/G $G^{\Delta_{m}}$. Thus, we have verified that the maximal chain of $G$-blocks $\{\alpha\}=\Delta_{0} \subset \ldots \subset \Delta_{m}$ satisfies the conditions of Part(3) of Theorem 3.5 implying that $G$ is nilpotent. Putting it all together we have the following theorem.

Theorem 3.9. There is a polynomial-time algorithm that takes $f \in \mathbb{Q}[X]$ as input and tests if $\mathrm{Gal}(f)$ is nilpotent.

\section{Generalizing the Landau-Miller solvability test}

In this section we show that the Landau-Miller solvability test can be adapted to test if the Galois group of $f(X) \in \mathbb{Q}[X]$ is in $\Gamma_{d}$ for constant $d$. Note that for $d<5, \Gamma_{d}$ is the class of solvable groups and hence our result is a generalization of the result of Landau-Miller [4. We first recall a well-known bound on the size of primitive permutation groups in $\Gamma_{d}$.

Theorem 4.1 ([1]). Let $G \leq S_{n}$ be a primitive permutation group in $\Gamma_{d}$ for a constant d. Then $\# G \leq n^{O(d)}$. 
Theorem 4.2. For constant $d>0$, there is an algorithm that takes as input $f(X) \in \mathbb{Q}[X]$ and in time polynomial in size $(f)$ and $n^{O(d)}$ decides whether $\operatorname{Gal}(f)$ is in $\Gamma_{d}$.

Proof. We sketch the proof. Assume without loss of generality that $f(X)$ is irreducible. Let $G=\operatorname{Gal}(f)$ as a subgroup of $\operatorname{Sym}(\Omega)$, where $\Omega$ is the set of roots of $f$. Let $\{\alpha\}=\Delta_{0} \subset \ldots \subset \Delta_{t}=\Omega$ be any maximal chain of $G$-blocks. The series $\{1\}=G^{\Delta_{0}} \triangleleft \ldots \triangleleft G^{\Delta_{t}}=G$ gives a normal series for $G$. By closure properties of $\Gamma_{d}, G \in \Gamma_{d}$ iff $\frac{G^{\Delta_{i+1}}}{G^{\Delta_{i}}} \in \Gamma_{d}$ for each $i$. If $G$ is in $\Gamma_{d}$ so are $G_{\Delta_{i+1}}$ and $\mathrm{G}\left(\Delta_{i+1} / \Delta_{i}\right)$ and hence their quotient $\frac{G_{\Delta_{i+1}}}{\mathrm{G}\left(\Delta_{i+1} / \Delta_{i}\right)}$. On the other hand since $\frac{G^{\Delta_{i+1}}}{G^{\Delta_{i}}}$ is isomorphic to a subgroup of $\left(\frac{G_{\Delta_{i+1}}}{G\left(\Delta_{i+1} / \Delta_{i}\right)}\right)^{l}$ for some $l$ (Lemma 2.3, $\frac{G^{\Delta_{i+1}}}{G^{\Delta_{i}}} \in \Gamma_{d}$ if $\frac{G_{\Delta_{i+1}}}{\mathrm{G}\left(\Delta_{i+1} / \Delta_{i}\right)} \in \Gamma_{d}$. Hence $G \in \Gamma_{d}$ iff $\frac{G_{\Delta_{i+1}}}{\mathrm{G}\left(\Delta_{i+1} / \Delta_{i}\right)}$ is in $\Gamma_{d}$ for each $i$. We give a polynomial-time algorithm to verify the above fact for some maximal chain of $G$-blocks $\{\alpha\}=\Delta_{0} \subset \ldots \subset \Delta_{t}=\Omega$.

First, by Theorem 3.7 we compute $K_{i}=\mathbb{Q}_{\Delta_{i}}$ for a maximal chain of $G$ blocks $\{\alpha\}=\Delta_{0} \subset \ldots \subset \Delta_{t}=\Omega$. Let $L_{i}$ be the fixed field of $\mathbb{Q}_{f}$ with respect to the automorphisms of $\mathrm{G}\left(\Delta_{i+1} / \Delta_{i}\right)$ then $L_{i+1}$ is the normal closure of $K_{i}$ over $K_{i+1}$. This follows because $\mathrm{G}\left(\Delta_{i+1} / \Delta_{i}\right)$ is the largest proper normal subgroup of $G_{\Delta_{i+1}}=\operatorname{Gal}\left(\mathbb{Q}_{f} / \mathbb{Q}_{\Delta_{i+1}}\right)$. Hence $\operatorname{Gal}\left(L_{i+1} / K_{i+1}\right)$ is $\frac{G_{\Delta_{i+1}}}{\mathrm{G}\left(\Delta_{i+1} / \Delta_{i}\right)}$, and it suffices to check that each $\operatorname{Gal}\left(L_{i} / K_{i}\right)$ is in $\Gamma_{d}$.

The group $\frac{G_{\Delta_{i+1}}}{\mathrm{G}\left(\Delta_{i+1} / \Delta_{i}\right)}$ acts faithfully and primitively on $\Omega^{\prime}=\mathcal{B}\left(\Delta_{i+1} / \Delta_{i}\right)$, by Lemma 2.3 and since $\Delta_{i}$ is a maximal subblock of $\Delta_{i+1}$. If $G \in \Gamma_{d}$ then $\left[L_{i+1}: K_{i+1}\right]=\# \operatorname{Gal}\left(L_{i+1} / K_{i+1}\right) \leq n^{O(d)}$ and degrees $\left[L_{i}: \mathbb{Q}\right]$ are all less than $n^{O(d)}$. We can use Theorem 1.2 to compute $\operatorname{Gal}\left(L_{i} / K_{i}\right)$ as a multiplication table in time polynomial in size $(f)$ and $n^{d}$ for each $i$. We then verify that Gal $\left(L_{i} / K_{i}\right) \in \Gamma_{d}$ by computing a composition series for it and checking that each composition factor is in $\Gamma_{d}$. At any stage in the computation of $\operatorname{Gal}\left(L_{i} / K_{i}\right)$ if the sizes of the fields becomes too large, i.e. larger than the bound of Theorem 4.1 we abort the computation and decide that $\operatorname{Gal}(f)$ is not in $\Gamma_{d}$. Clearly, these steps can be done in polynomial time.

It follows from the proof of Theorem 4.2 that a prime $p$ divides \#Gal $(f)$ if and only if it divides $\left[L_{i}: K_{i}\right]$ for some $1 \leq i \leq t$.

Theorem 4.3. Given $f(X) \in \mathbb{Q}[X]$ with Galois group in $\Gamma_{d}$ there is an algorithm running in time polynomial in size $(f)$ and $n^{d}$ that computes all the prime factors of $\# \operatorname{Gal}(f)$.

\section{References}

[1] L. Babai, P. J. Cameron, and P. P. Pálfy. On the order of primitive groups with restricted nonabelian composition factors. Journal of Algebra, 79:161$168,1982$. 
[2] P. Fernandez-Ferreiros and M. A. Gomez-Molleda. Deciding the nilpotency of the galois group by computing elements in the centre. Mathematics of Computation, 73(248), 2003.

[3] S. Landau. Polynomial time algorithms for galois groups. In J. Fitch, editor, EUROSAM 84 Proceedings of International Symposium on Symbolic and Algebraic Computation, volume 174 of Lecture Notes in Computer Sciences, pages 225-236. Springer, July 1984.

[4] S. Landau and G. L. Miller. Solvability by radicals is in polynomial time. Journal of Computer and System Sciences, 30:179-208, 1985.

[5] S. Lang. Algebra. Addison-Wesley Publishing Company, Inc, third edition, 1999.

[6] H. W. Lenstra Jr. Algorithms in algebraic number theory. Bulletin of the American Mathematical Society, 26(2):211-244, April 1992.

[7] E. M. Luks. Isomorphism of graphs of bounded valence can be tested in polynomial time. Journal of Computer and System Sciences, 25(1):42-65, 1982.

[8] E. M. Luks. Permutation groups and polynomial time computations. DIMACS Series in Discrete Mathematics and Theoretical Computer Science, 11:139-175, 1993.

[9] H. Wielandt. Finite Permutation Groups. Academic Press, New York, 1964. 